\title{
STRESS EM ESTUDANTES DE GRADUAÇÃO EM UMA UNIVERSIDADE PÚBLICA DA REGIÃO NORTE DE MOÇAMBIQUE
}

Stress in graduate students in Public University of the northern of Mozambique

Stress des étudiants gradués dans une Université Publique au Nord du Mozambique

Estrés en estudiantes graduados de uma Universidad Pública de la Región Mozambique del Norte

Gildo Aliante ${ }^{1}$

ORCID: http://orcid.org/0000-0002-6283-9544 Universidade Federal do Rio Grande do Sul, Porto Alegre, Rio Grande do Sul, Brasil. Doutorando do PPG em Psicologia Social e Institucional da Universidade Federal do Rio Grande do Sul - Brasil. Mestre em Psicologia Social e Institucional pela UFRGS. Graduado (Bacharelato e Licenciatura) em Planificação, Administração e Gestão da Educação pela Universidade Pedagógica - Moçambique. Membro do Núcleo de Estudos e Pesquisa em Saúde e Trabalho (NEST/UFRGS). Pesquisador do Grupo de Estudos em Saúde e Trabalho (GEST) do Laboratório de Psicologia da UniRovuma - Nampula. Bolsista de CAPES

Mussa Abacar ${ }^{2}$

ORCID: http://orcid.org/0000-0001-7797-8101 Universidade Rovuma, Nampula, Moçambique.

Faculdade Educação e Psicologia na Universidade Rovuma - Nampula. Doutor em Psicologia Cognitiva pela Universidade Federal de Pernambuco-Brasil. Mestre em Psicologia das Organizações, Social e do Trabalho pela

Universidade do Porto - Portugal. Licenciado em Psicologia e Pedagogia pela Universidade Pedagógica Moçambique. Coordenador do Grupo de Estudos em Saúde e Trabalho (GEST) do Laboratório de Psicologia da UniRovuma - Nampula

Bonciano Hilário Saquina ${ }^{3}$

ORCID: http://orcid.org/0000-0002-4426-3799 Universidade Rovuma, Nampula, Moçambique. Faculdade de Educação e Psicologia na Universidade Rovuma - Nampula. Mestrando em Administração e Gestão da Educação pela Universidade Pedagógica - Moçambique. Licenciado em Psicologia e Pedagogia pela

Universidade Pedagógica - Moçambique. Membro do Grupo de Estudos em Saúde e Trabalho (GEST) do Laboratório de Psicologia da UniRovuma - Nampula

ORCID: http://orci Carlos Luís Aristides ${ }^{4}$

Escola Primaria de Namutequeliua, Nampula, Moçambique. Graduado (Licenciatura) em Psicologia Social e das Organizações com Habilitações em Comportamento Organizacional pela Universidade Rovuma - Moçambique. Membro do Grupo de Estudos em Saúde e Trabalho (GEST) do Laboratório de Psicologia na UniRovuma - Nampula.

1 aliantegildo@yahoo.com.br

2 abacarmussa@yahoo.com.br

${ }^{3}$ boncianosaquina@gmail.com

${ }^{4}$ carlosluisaritides93@gmail.com

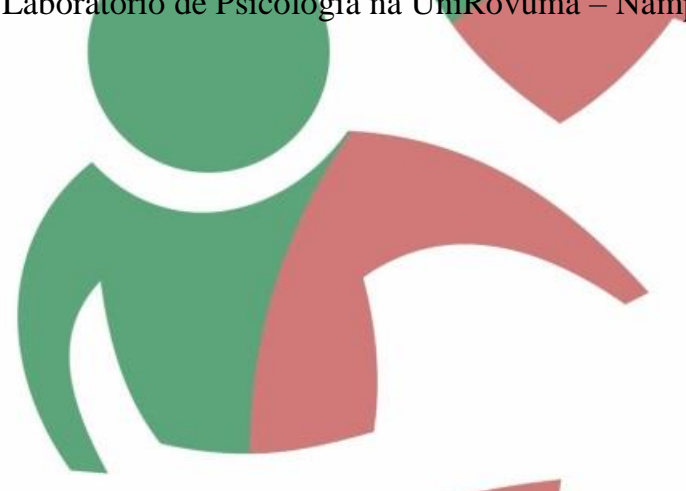




\title{
Resumo
}

O dia-a-dia do estudante no ensino superior é marcado por múltiplas exigências, próprias da vida acadêmica, o que pode ocasionar em certos casos o aparecimento de stress. Este estudo objetivou avaliar a sintomatologia de stress em estudantes de graduação em uma universidade pública da região norte de Moçambique. A pesquisa envolveu uma amostra de 176 estudantes, sendo 107 do sexo masculino e os restantes 69 do sexo feminino, com a faixa etária entre 17 a 53 anos. Os dados foram coletados por meio de um questionário sociodemográfico, Inventário de Sintomas de Stress de Lipp e analisados com auxílio do pacote estatístico SPSS, versão 25. Os resultados revelaram que $154(87,5 \%)$ participantes apresentaram sintomas de stress, dos quais $112(63,6 \%)$ na fase de resistência, $33(18,75 \%)$ de alerta e $9(5,15 \%)$ de exaustão, com sintomatologia predominantemente de natureza psicológica. O perfil de estudantes com maior vulnerabilidade à doença é: ser do sexo masculino, mais novo, solteiro, estar no início do curso, estudar no regime diurno e não possuir vínculo empregatício. Esses achados salientam a necessidade de realização de mais estudos de modo a aprimorar a compreensão do fenômeno de stress nos estudantes e fatores associados, bem como o desenvolvimento de ações preventivas e interventivas.

Palavras-chave: Stress; Saúde mental; Ensino superior; Bem-estar psicológico.

\begin{abstract}
The daily life of students in higher education is marked by multiple demands, typical of academic life, which can in some cases lead to the emergence of stress. This study aimed to evaluate stress symptoms in undergraduate students at a public university in northern Mozambique. The survey involved a sample of 176 students, 107 males and the remaining 69 females, aged 17 to 53 years. Data were collected through a sociodemographic questionnaire, Lipp Stress Symptom Inventory and analyzed using the SPSS version 25 statistical package. The results revealed that $154(87.5 \%)$ participants had symptoms of stress, of which $112(63,6 \%)$ in the resistance phase, $33(18,75 \%)$ of alertness and $9(5,15 \%)$ of exhaustion, with predominantly psychological symptoms. The profile of students with greater vulnerability to the disease is: to be male, younger, single, to be at the beginning of the course, to study in the daytime regime and not to have employment relationship. These findings highlight the need for further studies to improve understanding of the stress phenomenon in students and associated factors, as well as the development of preventive and interventional actions.
\end{abstract}

Keywords: Stress; Mental health; Higher education; Psychological well-being.

\section{Résumé}

Le quotidien de l'étudiant dans l'enseignementsupérieur est marqué par des exigences multiples, propres de la vie académique et dans certaines situations cela peut entrainer le développement du stress. Cette étude a le but d'évaluer la symptomatologie du stress chez lesétudiants gradués dans l'une desuniversités publiques au nord du Mozambique. L'échantillon de notre recherche est composé de 176 étudiants âgés de 17 à 53 ans dont 107 du sexe masculin et les autres 69 du sexe féminin. Pour la collecte des données nous avons utilisé un questionnaire sociodémographique et l'inventaire de Symptômes de Stress de Lipp analysé à travers le logicielstatistique SPSS, $25^{\mathrm{e}}$ version.. Les résultats de l'analyse indiquent la présence des symptômes du stress chez $154(87.5 \%)$ des participants, dont $112(63,6 \%)$ en état de résistance, $33(18,75 \%)$ en état d'alerte et $9(5,15 \%)$ en étatd'épuisement, avec une symptomatologie essentiellement psychologique. En termes de facteurs de vulnérabilité, cette maladie affecte principalement des étudiantsdu sexe masculin, les plus jeunes, célibataires, en début d'un cursus, ayant cours dans la période de la journée et en situation de chômage. Cette maladie est plus frequente dans les étudiants du sexe masculin entre autres nous contactons les raisons suivantes être le plus jeune, être en première année du cours, être celibataire, être dans le régime régulier et le problème du chomage. Nous pensons avoir besoin de réaliser plus d'études de façon à mieux comprendre le phénomènedustress chez les étudiants, ses facteurs au, en vue à la mise en place d'actions de prévention et d'intervention.

Mots clés: Stress; Santé mentale; Enseignement Supérieur; Bien être psicologique

\section{Resumen}

La vida diaria de los estudiantes en la educación superior está marcada por múltiples demandas, típicas de la vida académica, que en algunos casos pueden conducir a la aparición de estrés. Este estudio tuvo como objetivo evaluar los síntomas del estrés en estudiantes universitarios de una universidad pública en el norte de Mozambique. La 
encuesta incluyó una muestra de 176 estudiantes, 107 hombres, y lo restantes, 69 mujeres, de 17 a 53 años. Los datos fueron recolectados a través de un cuestionario sociodemográfico, Lipp Stress Symptom Inventory y analizados utilizando el paquete estadístico SPSS versión 25. Los resultados revelaron que $154(87,5 \%)$ participantes tenían síntomas de estrés, de los cuales 112 (63.6\%) en la fase de resistencia, 33 (18,75\%) de alerta y $9(5,15 \%)$ de agotamiento, con síntomas predominantemente psicológicos. El perfil de los estudiantes con mayor vulnerabilidad a la enfermedad es ser hombres, jóvenes, solteros, estar al comienzo del curso, estudiar en el régimen diurno y no tener una relación laboral. Estos hallazgos resaltan la necesidad de más estudios para mejorar la comprensión del fenómeno del estrés en los estudiantes y los factores asociados, así como el desarrollo de acciones preventivas e intervencionistas.

Palabras clave: Estrés; Salud mental; Enseñanza superior; Bienestar psicológico.

\section{INTRODUÇÃO}

O ingresso na Universidade constitui um importante marco na vida do estudante que inicia um universo acadêmico repleto de normas, metodologias, grupos e pessoas desconhecidas. Essa nova realidade educacional cria a necessidade do estudante desenvolver um perfil universitário, pois, com todas as mudanças características desta etapa, novas demandas são geradas e este tem que se adaptar a esta nova realidade (Castro, 2017). Este processo pode, por vezes, ser percebido como um stressor e impactar diretamente na saúde dos estudantes (Ariño \& Bardagi, 2018). Durante a fase de vida universitária, os estudantes deparam-se com diversos stressores tais como: falta de tempo para atividades de lazer, incertezas quanto ao futuro profissional, carga horária elevada do curso, volume elevado de matérias para estudar, números de horas de sono insuficientes, dificuldade na gestão do tempo, dificuldades de ensino/aprendizagem (Santos et al., 2012), o distanciamento da casa dos familiares, a adaptação ao ambiente universitário; a sobrecarga de tarefas, os conflitos interpessoais, baixo rendimento acadêmico, dificuldade de lidar com as matérias (Araújo et al., 2016), a falta de tempo para estar com amigos e para descansar, falta de competência, más relações interpessoais com supervisor e colegas, sobrecarga, impotência e incerteza (Martins, Campos, Duarte, Martins, Moreira, \& Chaves, 2017), além da realização de provas e trabalhos de aula, sentimentos de perfeccionismo e auto-exigência (Chagas, Moreira Junior, Cunha, Caixeta, \& Fonseca, 2016).

Um estudo realizado por Salomão, Abacar e Aliante (2018), envolvendo uma amostra de estudantes de ensino superior em Moçambique, revelou que as variáveis "número de disciplinas reprovadas", "ano de frequência", "número de vezes que o aluno reprovou", correlacionaram-se negativamente com a satisfação discente. Além do mais, os alunos pesquisados apontaram as condições físicas (falta de higiene nas salas de aulas e banheiros, insuficiência de salas, falta de equipamento audiovisual e ventiladores nas salas de aula); fraca 
preparação acadêmica dos professores, falta de domínio da língua inglesa e as práticas de avaliação como aspectos críticos e percebidos pelos estudantes pesquisados como stressores da vida acadêmica.

O stress, um conjunto de reações que ocorrem no organismo quando submetido a um esforço de adaptação (Seyle,1936), envolve quatro fases, nomeadamente: a fase de alarme, a fase de resistência, a fase de quase-exaustão e a fase de exaustão (Lipp, 2016). A fase de alarme é caracteriza-se pelo início de mudanças corporais no indivíduo face à um agente indutor de stress que o aflige, sendo as alterações que se presenciam particularmente mediadas pela ativação do sistema simpático-medular, permitindo ao indivíduo estar preparado para lutar ou fugir da situação estressora. Esta fase é assinada pelo aumento da frequência cardíaca e respiratória, da subida de tensão arterial e do vaso constrição, da região esplâncnica e dos membros (Ramos \& Carvalho, 2008), contração de baço, levando mais glóbulos vermelhos à corrente sanguínea, acarretando mais oxigênio para o organismo (Limongi-França \& Rodrigues, 2009), ficando as extremidades frias e suadas. Esta fase termina quando o agente indutor do stress e caso contrário, o indivíduo entra na fase seguinte, a de resistência.

A fase de resistência caracteriza-se por um aumento de córtex supra-renal, do baço e de todas as estruturas linfáticas, do número de glóbulos sanguíneos, úlceras no sistema digestivo, aumento de concentração de cloro na corrente sanguínea, além dos sintomas como irritabilidade, insônia, mudanças de humor e diminuição do desejo sexual (Limongi-França \& Rodrigues, 2009). Se, nesta fase, o indivíduo conseguir lidar com a situação estressora, dá-se um apaziguamento geral dos sintomas, mas se o problema se mantém o indivíduo poderá passar para a fase posterior (Ramos \& Carvalho, 2008), a de quase-exaustão.

A fase quase-exaustão é caracterizada pelo enfraquecimento do organismo e início do processo da doença. Precisa-se, então, de ajuda tanto médica para tratar o problema físico que já surgiu, como psicológica para aprender a lidar com a causa do stress. Devido ao enfraquecimento do organismo, doenças que permaneciam latentes são desencadeadas. Caso o stressor permaneça presente e a pessoa não aprenda a lidar com ele adequadamente, ocorrerá a fase de exaustão (Lipp, 2016).

A quarta fase, a de exaustão, representa muitas vezes a falha de mecanismo de adaptação. Nesta fase, ocorrem doenças graves e a pessoa não consegue mais recuperar sozinha, não consegue se concentrar, pensar direito, rir, ter gosto pela vida,entra em depressão e aparecem várias doenças físicas graves. Isto resulta do stressor prolongado que afeta diretamente o sistema imunológico, reduzindo a resistência da pessoa e tornando-a vulnerável ao desenvolvimento de infecções e doenças contagiosas (Lipp, 2016). 
A prevalência do stress no mundo é alta e deveras preocupante. Estima-se que o stress, enquanto problema global esteja a afetar um em cada três trabalhadores do mundo inteiro, sendo que o ritmo das mudanças que operam nos contextos modernos de trabalho pode propiciar um provável agravamento da doença, caso não sejam realizados esforços visando àprevenção (Murphy, 2008). É assim que várias organizações internacionais estão promovendo reuniões mundiais para a discussão de medidas preventivas que possam ser tomadas de modo a reluzi-lo (Lipp, Costa, \& Nunes, 2017). Estas autoras advogam ainda que a International Labour Organization (ILO), em 2016, realizou uma pesquisa com 324 especialistas de 54 países de várias partes do mundo, concluindo que o stress é um problema global, uma vez que mais de 90\% dos participantes o indicaram como uma real preocupação nos seus países.

O stress não é apenas um problema que afeta o trabalhador. Ao nível do ensino superior, por exemplo, as exigências impostas aos estudantes e as dificuldades por estes encaradas podem desencadear stress como revelaram alguns estudos (e.g., Assis, Silva, Lopes, Silva, \& Santani, 2013; Cestari, Barbosa, Florêncio, Pessoa, \& Moreira, 2017; Lima et al., 2016; Querido, Naghettini, Orsini, Bartholomeu, \& Montie, 2016; 2016; Vieira \& Schermann, 2015) e demais problemas de saúde mental como a depressão e ansiedade. Alguns autores (e.g., Chagas et al., 2016; Querido et al., 2016; Hirsch, Barlem, Almeida, Tomaschewski-Barlem, Lunardi, \& Ramos, 2018; Lima, Soares, Prado, \& Albuquerque, 2016; Martins et al., 2017) listam os seguintes fatores associados ao stress em estudantes: esquema de estudo, provas e exames, gestão do tempo, conflitos entre dever e lazer, sentimento de desamparo frente ao poder dos professores, constante competição entre os estudantes; divisão dos alunos em grupos, dilemas éticos nas situações de alta complexidade, falta de tempo para lazer e estar com amigos, mau relacionamento interpessoal, preocupações quanto à absorção das informações ao longo do curso e preocupação com os ganhos financeiros no futuro.

Percebe-se que quando um fenômeno como o stress se torna tão preyalente, a busca pela identificação dos seus sintomas, causas e seus efeitos, torna-se relevante, de modo a apoiar a planejar programas preventivos, sendo um dos motivos que justifica a realização desta pesquisa. Apesar disso, a temática de stress em estudantes é tão precariamente discutida no contexto moçambicano.

O objetivo deste estudo foi de avaliar a sintomatologia do stress em estudantes de graduaçãoem uma universidade pública da região norte de Moçambique. Em função desse objetivo, duas questões nortearam este estudo, a saber: a) quais são os principais sintomas de stress presentes nos estudantes de cursos de graduação em uma instituição de ensino superior 
moçambicana pública, em Nampula? b) qual é a frequência dos sintomas de stress em função das variáveis sociodemográficas e acadêmicas?

\section{MÉTODO}

Este estudo é transversal, descritivo e de natureza quantitativa. Foi desenvolvido em um campus de uma universidade pública localizada na cidade de Nampula, em Moçambique. O estudo envolveu uma amostra não probabilística por acessibilidade de 176 estudantes dos cursos de licenciatura em ensino de Educação Física e Desporto, Matemática, Física, Psicologia Social e das Organizações. Primeiramente foi solicitada uma autorização para a realização da pesquisa junto da Direção de Pós-graduação, Pesquisa e Extensão da Universidade por meio de uma carta de que constavam os objetivos, a metodologia e relevância social e cientifica, respondida favoravelmente. Posteriormente, foram contatadas as coordenações dos cursos que também foram informadas sobre as intenções da pesquisa e respectivos procedimentos metodológicos. E finalmente, os coordenadores acompanharam os pesquisadores nas turmas, em que decorreu o preenchimento por escrito dos questionários e de forma individual. Igualmente, os participantes foram informados sobre as pretensões da pesquisa, respectivos benefícios e riscos em participar da mesma.

Os alunos que consentiram em participar da pesquisa receberam as instruções dadas pelo terceiro e quarto autor. Foram cumpridos todos os procedimentos éticos vigentes em Moçambique e na Universidade Rovuma, nomeadamente o consentimento livre e esclarecido, o anonimato dos participantes, a confidencialidade dos dados e a proteção dos sujeitos de possíveis danos psicológicos. Caso algum participante apresentasse sintomas de stress e/ou se sentisse desconfortável durante a realização da pesquisa, teria o devido acompanhamento da equipe de coleta de dados composta por dois psicólogos. E, em caso grave, seria encaminhado ao Grupo de Estudos em Saúde e Trabalho (GEST) do Laboratório de Psicologia da UniRovuma, que é coordenado pelo segundo autor que também tem formação em Psicologia.

A coleta de dados foi feita em salas de aulas, nos meses de junho e julho de 2019. Os dados foram coletados por meio de um questionário de dados sociodemográficos e acadêmicos e o Inventario de Sintomas de Stress para Adultos de Lipp (ISSL). O questionário de dados sociodemográficos tinha objetivo de coletar informações pessoais e acadêmicas, especificamente: idade, sexo, curso, ano de frequência, informações que foram usadas para determinar o perfil dos participantes. 
O Inventário de Sintomas de Stress para Adultos de Lipp (ISSL) é um instrumento de avaliação do stress, validado e padronizado por Lipp e Guevara em 1994 e publicado pela casa do psicólogo (Lipp, 2000). Este instrumento visa identificara sintomatologia que o indivíduo apresenta, bem como em que fase de stress o indivíduo se encontra, de acordo com o modelo quadrifásico do stress de Lipp (alerta, resistência, quase-exaustão e exaustão). Também é observado o tipo de sintoma predominante, o físico ou o psicológico (Faiad, Souza, Matsunaga, Rodrigues, \& Rosa, 2018; Lipp et al., 2017).

O ISSL é constituído por 53 itens, dos quais 34 são referentes aos sintomas físicos e 19 aos psicológicos, dividindo-se em três partes. A primeira parte indica sintomas sentidos nas últimas24horas e é composta por 15 sintomas, 12 sintomas físicos e três psicológicos. $\mathrm{Na}$ segunda parte são apontados sintomas experimentados na última semana, sendo 10 físicos e cinco psicológicos. Finalmente, a terceira e última parte que abrange sintomas sentidos no último mês, composto por 12 físicos e 11 psicológicos. A avaliação das respostas dadas pelos indivíduos é feita por meio do uso de tabelas do próprio manual ISSL que transformam os dados brutos em porcentagem (Lipp et al., 2017; Souza, Santana, Pedra, Dias, Henrique, \& Dantas, 2015).

O respondente é solicitado a indicar se tem apresentado o sintoma de stress especificado em cada quadro em 24 horas (Q1-parte um), uma semana (Q2-parte dois) ou um mês (Q3-parte três). A avaliação é feita em termos das tabelas porcentuais do teste. A presença de stress pode ser constatada se qualquer um dos escores brutos atingir os limites determinados (maior que seis no Q1- parte um, ou seja a soma de quantidade de sintomas de F1 e P1; maior que três no Q2- parte dois, soma de F2 e P2 e maior que oito no Q3-parte três soma de F3 e P3). Caso os escores obtidos estejam acima dos limites nos três quadros, é identificado o processo de agravamento do stress. Para definir a fase de stress em que o indivíduo se encontra, deve-se considerar o quadro em que o mesmo mais pontuou, em termos porcentuais, sendo que os seus resultados permitem saber em que fase do stress a pessoa se encontra e se os sintomas que prevalecem são mais físicos ou psicológicos (Faiad et al., 2018).

$\mathrm{Na}$ sua versão original com amostras diversificadas, incluindo estudantes de ensino superior, o ISSL apresentou alfa de Cronbach de 0,91 para a escala geral, portanto os itens refletem um alto valor para o conceito intencional, qual seja: medir o nível de stress (Chaves et al., 2016; Lipp et al., 2017). Além de o ISSL revelar uma boa consistência interna, a escolha desse instrumento justifica-se por ser uma ferramenta mais utilizada na medição dos sintomas de stress (Souza et al., 2015), bem como ser um instrumento redigido em língua portuguesa. Assim, embora reconhecer algumas particularidades entre o português brasileiro e o falado em 
Moçambique, considerou-se o ISSL como ideal para identificar os sintomas de stress em estudantes moçambicanos.

A análise dos dados foi com base no software estatístico SPSS (Statistical Package for Social Sciences), versão 25. E para determinar o perfil dos participantes e identificar os sintomas de stress, foi utilizada a abordagem estatística descritiva, observando as frequências das respostas nos itens da escala para a elaboração das tabelas. Foram calculadas as frequências (absolutas e relativas) para determinar os sintomas mais regulares e as respectivas fases prevalecentes que o instrumento compõe.

\section{RESULTADOS}

\section{Perfil dos participantes}

Este estudo envolveu 176 estudantes que estudam em uma Instituição do Ensino Superior público, dos quais $107(60,8 \%)$ eram do sexo masculino e 69 (39.2\%) do sexo feminino, com a média deidade de 32 anos, numa variância de 17 a 55 anos. No que diz respeito ao estado civil, 158 (89,8\%) eram solteiros e 18 (10.2\%) declararam ser casados e/ou vivem em união estável. Do total de participantes, $82(46,6 \%)$ cursavam o curso de Licenciatura Psicologia Social e das Organizações, 44 (25\%) o de Licenciatura Educação Física e Desporto, $32(18,2 \%)$ frequentavam o curso de Licenciatura em Ensino de Física e 18 (10,2\%) cursavam a Licenciatura em Ensino de Matemática. Dos 176 participantes, 97 (55,1\%) frequentava o primeiro ano e $79(44,9 \%)$ o quarto ano. A maioria dos participantes, em número de 140 $(79,5 \%)$ estudava no regime diurno e 36 (20,5\%) no noturno. Em relação ao vínculo empregatício, $116(65,9 \%)$ não possuía qualquer vínculo laboral e $60(34,1 \%)$ tinha emprego.

\section{Frequência dos sintomas de stress}

Para identificar os sintomas mais regulares na fase de alerta, resistência e exaustão foi feito o cálculo das frequências (absolutas e relativas) dos sintomas expostos no ISSL, como ilustra a Tabela 1 . 
Tabela 1: Frequência dos sintomas físicos e psicológicos nos estudantes $(n=176)$

\begin{tabular}{|c|c|c|}
\hline \multicolumn{3}{|l|}{ Fase de alarme } \\
\hline Sintomas físicos (F1) & $\mathrm{n}$ & $\%$ \\
\hline Mãos e pés frios & 74 & 42,0 \\
\hline Boca seca & 45 & 25,6 \\
\hline Nó no estomago & 19 & 10,8 \\
\hline Aumento de sudorese & 2 & 1,1 \\
\hline Tensão muscular & 11 & 6,3 \\
\hline Aperto de mandíbula/ranger de dentes & 13 & 7,4 \\
\hline Diarréia passageira & 4 & 2,3 \\
\hline Insônia & 12 & 6,8 \\
\hline Taquicardia & 2 & 1,1 \\
\hline Hiperventilação & 4 & 2,2 \\
\hline Hipertensão arterial súbita e passageira & 2 & 1,1 \\
\hline Mudança de apetite & 7 & 4,0 \\
\hline Sintomas psicológicos (P1) & $\mathrm{n}$ & $\%$ \\
\hline Aumento súbito de motivação & 93 & 52,8 \\
\hline Vontade súbita de iniciar novos projetos & 48 & 27,3 \\
\hline Entusiasmo súbito & 35 & 19,9 \\
\hline \multicolumn{3}{|l|}{ Fase de resistência } \\
\hline Sintomas físicos (F2) & $\mathrm{n}$ & $\%$ \\
\hline Problemas com a memória & 86 & 48,9 \\
\hline Mal-estar generalizado, sem causa específica & 44 & 25,0 \\
\hline Formigamento nas extremidades & 7 & 4,0 \\
\hline Sensação de desgaste físico constante & 10 & 5,7 \\
\hline Mudança no apetite & 20 & 11,4 \\
\hline Aparecimento de problemas dermatológicos & 13 & 7,4 \\
\hline Hipertensão arterial & 3 & 1,7 \\
\hline Cansaço constante & 100 & 56,8 \\
\hline Gastrite, úlcera ou indisposição estomacal muito prolong: & 1 & 0,6 \\
\hline Tontura ou sensação de estar flutuando & 1 & 0,6 \\
\hline Sintomas psicológicos (P2) & $\mathrm{n}$ & $\%$ \\
\hline Sensibilidade emotiva excessiva & 37 & 21,0 \\
\hline Dúvida quanto a si próprio & 80 & 45,5 \\
\hline Pensar constantemente em um só assunto & 36 & 20,5 \\
\hline Irritação excessiva & 22 & 12,5 \\
\hline Diminuição da libido & 1 & 0,6 \\
\hline \multicolumn{3}{|l|}{ Fase de quase exaustão e exaustão } \\
\hline Sintomas físicos (F3) & $\mathrm{n}$ & $\%$ \\
\hline Diarreia frequente & 14 & 8,0 \\
\hline Dificuldades sexuais & 35 & 19,9 \\
\hline Insônia & 58 & 33,0 \\
\hline Náusea & 15 & 8,5 \\
\hline Tiques & 4 & 2,3 \\
\hline Hipertensão arterial continuada & 7 & 4,0 \\
\hline Problemas dermatológicos prolongados & 7 & 4,0 \\
\hline
\end{tabular}


Mudança extrema de apetite

Excesso de gases

Tontura frequente

Úlcera, colite ou outro problema digestivo sério

Infarto

Sintomas psicológicos (P3)

Impossibilidade de trabalhar

Pesadelos frequentes

Sensação de incompetência em todas as áreas

Apatia, depressão ou raiva prolongada

Cansaço constante excessivo

Pensar e falar constantemente em um só assunto

Irritabilidade frequente sem causa aparente

Angústia, ansiedade, medo, diariamente

Perda de senso de humor

Hipersensibilidade emotiva
27

5

15,3

2,8

1,7

9,6

0,6

1

n

39

53

22,2

14

30,1

8,0

2,8

5

19

10,8

$20 \quad 11,4$

$2 \quad 1,1$

$2 \quad 1,1$

$10 \quad 5,6$

2

Fonte: Autores

A Tabela 1 ilustra que os sintomas físicos da fase de alerta mais apontados foram: mãos e pés frios e boca seca. Nos sintomas psicológicos, os mais evidenciados foram aumento súbito de motivação e vontade súbita de iniciar novos projetos. Enquanto para fase de resistência, os sintomas físicos mais indicados são cansaço constante, problemas com a memória, mal-estar generalizado e sem causa especifica. E nos sintomas psicológicos, o mais referenciado foi o de dúvida quanto a si próprio. $\mathrm{Na}$ fase de exaustão, o sintoma físico mais apontado é a insônia. $\mathrm{E}$ no quadro dos sintomas psicológicos, pesadelos frequentes é que obtive maior frequência. Em termos gerais, os sintomas mais regulares foram das fases de alerta e resistência, com quatro sinais dos 15 que compõe cada fase.

Frequência dos sintomas de stress em relação às variáveis acadêmicas.

Foi achada a frequência de sintomas de stress em função das variáveis sociodemográficas (sexo, estado civil e ano de frequência). Os resultados constam na tabela 2.

Tabela 2: Sintomas de stress em função das variáveis sociodemográficas (n=176)

\section{Sintomas}

Fase de alerta (Parte 1)
Variáveis Estado civil

Sexo

Masc. $\mathrm{n}(\%)$ sociodemográficas e

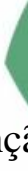

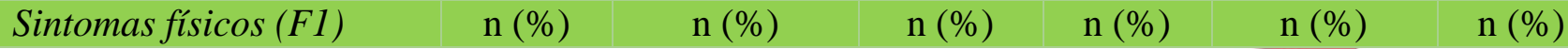




\begin{tabular}{|c|c|c|c|c|c|c|}
\hline Mãos e pés frios & $45(60,8)$ & $29(39,2)$ & $67(90,5)$ & $7(9,5)$ & $49(66,2)$ & $25(33,8)$ \\
\hline Boca seca & $31(68,9)$ & $14(31,1)$ & $43(95.6)$ & $2(4.4)$ & $25(55,6)$ & $20(44,4)$ \\
\hline Nó no estomago & $8(42,1)$ & $11(57,9)$ & $17(89,5)$ & $2(10,5)$ & $11(57,9)$ & $8(42,1)$ \\
\hline Aumento de sudorese & $2(100)$ & $0(0,0)$ & $2(100)$ & $0(0,0)$ & $0(0,0)$ & $2(100)$ \\
\hline Tensão muscular & $10(90,9)$ & $1(9,1)$ & $10(90,9)$ & $1(10,1)$ & $7(63,7)$ & $4(36,4)$ \\
\hline Diarreia passageira & $2(50)$ & $2(50)$ & $3(75)$ & $1(25)$ & $1(25)$ & $3(75)$ \\
\hline Insônia & $5(41,6)$ & $7(58.4)$ & $10(83.3)$ & $2(17.7)$ & $3(25)$ & $9(75)$ \\
\hline $\begin{array}{l}\text { Hipertensão arterial súbita } \\
\text { e passageira }\end{array}$ & $0(0,0)$ & $2(100)$ & $1(50)$ & $1(50)$ & $0(0,0)$ & $2(100)$ \\
\hline Mudança de apetite & $4(57,1)$ & $3(42,9)$ & $5(71,4)$ & $2(28,6)$ & $1(14,3)$ & $6(85,7)$ \\
\hline $\begin{array}{l}\text { Sintomas psicológicos } \\
(P 1)\end{array}$ & $\ldots \ldots \ldots \ldots$ & ……... & …............. & ….......... & ............... & …......... \\
\hline $\begin{array}{l}\text { Aumento súbito de } \\
\text { motivação }\end{array}$ & $58(62,4)$ & $35(37,6)$ & $82(88,2)$ & $11(11,8)$ & $60(61,9)$ & $33(41,8)$ \\
\hline Entusiasmo súbito & $21(60)$ & $14(40)$ & $33(94,3)$ & $2(5,7)$ & $21(60)$ & $14(40)$ \\
\hline $\begin{array}{l}\text { Vontade súbita de iniciar } \\
\text { novos projetos }\end{array}$ & $28(59.3)$ & $20(41.7)$ & $43(89.6)$ & $5(10.4)$ & $16(33,3)$ & $32(66,7)$ \\
\hline $\begin{array}{l}\text { Fase de resistência (Parte } \\
\text { 2) }\end{array}$ & Masculino & Femin. & Solteiro & Casado & $1^{\circ}$ ano & $4^{\circ}$ ano \\
\hline Sintomas físicos (F2) & $\mathrm{n}(\%)$ & $\mathrm{n}(\%)$ & $\mathrm{n}(\%)$ & $\mathrm{n}(\%)$ & $\mathrm{n}(\%)$ & $\mathrm{n}(\%)$ \\
\hline Problemas com a memória & $53(61,6)$ & $33(38,4)$ & $79(91,9)$ & $7(8,1)$ & $55(64)$ & $31(36)$ \\
\hline $\begin{array}{l}\text { Mal-estar generalizado, } \\
\text { sem causa especifica }\end{array}$ & $21(47,7)$ & $23(52,3)$ & $39(88,6)$ & $5(11,4)$ & $19(43,2)$ & $25(56,8)$ \\
\hline $\begin{array}{l}\text { Formigamento } \\
\text { extremidades }\end{array}$ & $4(57,1)$ & $3(42,9)$ & $6(85,7)$ & $1(14,3)$ & $5(71,4)$ & $2(28,6)$ \\
\hline $\begin{array}{l}\text { Sensação de desgaste } \\
\text { físico constante }\end{array}$ & $7(70)$ & $3(30)$ & $7(70)$ & $3(30)$ & $1(10)$ & $9(90)$ \\
\hline Mudança de apetite & $17(85)$ & $3(15)$ & $18(90)$ & $2(10)$ & $7(35)$ & $13(65)$ \\
\hline Hipertensão arterial & $2(66,7)$ & $1(33,3)$ & $3(100)$ & $0(0.0)$ & $1(33,3)$ & $2(66,7)$ \\
\hline Cansaço constante & $60(60)$ & $40(40)$ & $90(90)$ & $10(10)$ & $65(65)$ & $35(35)$ \\
\hline $\begin{array}{l}\text { Gastrite, ulcera ou } \\
\text { indisposição estomacal } \\
\text { muito prolongada }\end{array}$ & $0(0,0)$ & $1(100)$ & $1(100)$ & $0(0,0)$ & $1(100)$ & $0(0,0)$ \\
\hline $\begin{array}{l}\text { Tontura ou sensação de } \\
\text { estar flutuando }\end{array}$ & $1(100)$ & $0(0,0)$ & $1(100)$ & $0(0,0)$ & $1(100)$ & $0(0,0)$ \\
\hline $\begin{array}{l}\text { Sintomas psicológicos } \\
(P 2)\end{array}$ & ......... & ....... & .......... & ....... & ......... & ........ \\
\hline $\begin{array}{l}\text { Sensibilidade emotiva } \\
\text { excessiva }\end{array}$ & $24(64,9)$ & $13(35,1)$ & $33(89,2)$ & $4(10,8)$ & $23(62,2)$ & $14(37,8)$ \\
\hline Dúvida quanto a si próprio & $53(66,3)$ & $27(33,8)$ & $74(92,5)$ & $6(7,5)$ & $55(68,8)$ & $25(31,2)$ \\
\hline $\begin{array}{l}\text { Pensar constantemente em } \\
\text { um só assunto }\end{array}$ & $19(52,8)$ & $17(47,2)$ & $31(86,1)$ & $5(13,9)$ & $13(36,1)$ & $23(63,9)$ \\
\hline Irritação excessiva & $10(45,5)$ & $12(54,5)$ & $19(86,4)$ & $3(13,6)$ & $5(22,7)$ & $17(77,3)$ \\
\hline Diminuição da libido & 1(100) & $0(0,0)$ & $1(100)$ & $0(0,0)$ & $1(100)$ & $0(0.0)$ \\
\hline Fase de exaustão & ......... & $\ldots \ldots \ldots$ & ......... & .......... & ......... & $\ldots \ldots . .$. \\
\hline Sintomas físicos (F3) & $\mathrm{n}(\%)$ & $\mathrm{n}(\%)$ & $\mathrm{n}(\%)$ & $\mathrm{n}(\%)$ & $\mathrm{n}(\%)$ & $\mathrm{n}(\%)$ \\
\hline Diarreia frequente & $9(64,3)$ & $5(35,7)$ & $13(92,9)$ & $1(7,1)$ & $9(64,3)$ & $5(35,7)$ \\
\hline Dificuldades sexuais & $25(71,4)$ & $10(28,6)$ & $30(85,7)$ & $5(14,3)$ & $23(65,7)$ & $12(34,3)$ \\
\hline
\end{tabular}




\begin{tabular}{|c|c|c|c|c|c|c|}
\hline Insônia & $35(60,3)$ & $23(39,7)$ & $53(91,4)$ & $5(8,6)$ & $31(53,4)$ & $27(46,6)$ \\
\hline Náusea & $7(46,7)$ & $8(53,3)$ & $14(93,3)$ & $1(6,7)$ & $8(53,3)$ & $7(46,7)$ \\
\hline Tiques & $4(100)$ & $0(0,0)$ & $4(100)$ & $0(0,0)$ & $2(50)$ & $2(50)$ \\
\hline $\begin{array}{l}\text { Hipertensão } \\
\text { continuada }\end{array}$ & $1(14,3)$ & $6(85,7)$ & $3(42,9)$ & $4(57,1)$ & $0(0.0)$ & $7(100)$ \\
\hline $\begin{array}{l}\text { Problemas } \\
\text { dermatológicos } \\
\text { prolongados }\end{array}$ & $4(57,1)$ & $3(42,9)$ & $6(85,7)$ & $1(14,3)$ & $4(57,1)$ & $3(42,9)$ \\
\hline $\begin{array}{l}\text { Mudança extrema de } \\
\text { apetite }\end{array}$ & $16(59,3)$ & $11(40,7)$ & $26(96,3)$ & $1(3,7)$ & $15(55,6)$ & $12(44,4)$ \\
\hline Excesso de gases & $4(80)$ & $1(20)$ & $5(100)$ & $0(0,0)$ & $3(60)$ & $2(40)$ \\
\hline Tontura frequente & $1(33,3)$ & $2(66,7)$ & $3(100)$ & $0(0,0)$ & $2(66,7)$ & $1(33,3)$ \\
\hline Infarto & $1(100)$ & $0(0,0)$ & $1(100)$ & $0(0,0)$ & $0(0,0)$ & $1(100)$ \\
\hline $\begin{array}{l}\text { Sintomas psicológicos } \\
(P 3)\end{array}$ & (.......... & ......... & ........... & ........... & ........ & $\ldots \ldots$. \\
\hline $\begin{array}{l}\text { Impossibilidade de } \\
\text { trabalhar }\end{array}$ & $27(69,2)$ & $12(30,8)$ & $33(84,6)$ & $6(15,4)$ & $18(46,2)$ & $21(53,8)$ \\
\hline Pesadelos frequentes & $29(54,7)$ & $24(45,3)$ & $49(92,5)$ & $4(7,5)$ & $32(60,4)$ & $21(39,6)$ \\
\hline $\begin{array}{l}\text { Sensação } \\
\text { incompetência em todas } \\
\text { as áreas }\end{array}$ & $9(64,3)$ & $5(35,7)$ & $13(92,9)$ & $1(7,1)$ & $11(78,6)$ & $3(23,4)$ \\
\hline Vontade de fugir de tudo & $10(50)$ & $10(50)$ & $18(90)$ & $2(10)$ & $8(40)$ & $12(60)$ \\
\hline $\begin{array}{l}\text { Apatia, depressão ou raiva } \\
\text { prolongada }\end{array}$ & $2(40)$ & $3(60)$ & $4(80)$ & $1(20)$ & $2(40)$ & $3(60)$ \\
\hline $\begin{array}{ll}\text { Cansaço } & \text { constante } \\
\text { excessivo } & \end{array}$ & $12(63,2)$ & $7(36,8)$ & $18(94,7)$ & $1(5,3)$ & $12(63,2)$ & $7(36,8)$ \\
\hline $\begin{array}{l}\text { Pensar e falar } \\
\text { constantemente em um só } \\
\text { assunto }\end{array}$ & $12(60)$ & $8(40)$ & $17(85)$ & $3(15)$ & $10(50)$ & $10(50)$ \\
\hline $\begin{array}{l}\text { Irritabilidade frequente } \\
\text { sem causa aparente }\end{array}$ & $2(100)$ & $0(0,0)$ & $2(100)$ & $0(0.0)$ & $1(50)$ & $1(50)$ \\
\hline $\begin{array}{l}\text { Angustia, ansiedade e } \\
\text { medo diariamente }\end{array}$ & $2(100)$ & $0(0,0)$ & $2(100)$ & $0(0,0)$ & $2(100)$ & $0(0,0)$ \\
\hline $\begin{array}{l}\text { Hipersensibilidade } \\
\text { emotiva }\end{array}$ & $2(100)$ & $0(0,0)$ & $2(100)$ & $0(0,0)$ & $1(50)$ & $1(50)$ \\
\hline
\end{tabular}

Fonte: Autores

No seguimento das análises achou-se a frequência de sintomas de stress com as restantes variáveis de estudo. A Tabela 3 dá continuidade das frequências obtidas no cruzamento dos sintomas de stress com as variáveis "vínculo empregatício", "regime do curso" e "idade". 
Tabela 3: Frequência de sintomas de stress em função das variáveis "vínculo empregatício", "regime" e "idade" $(n=176)$

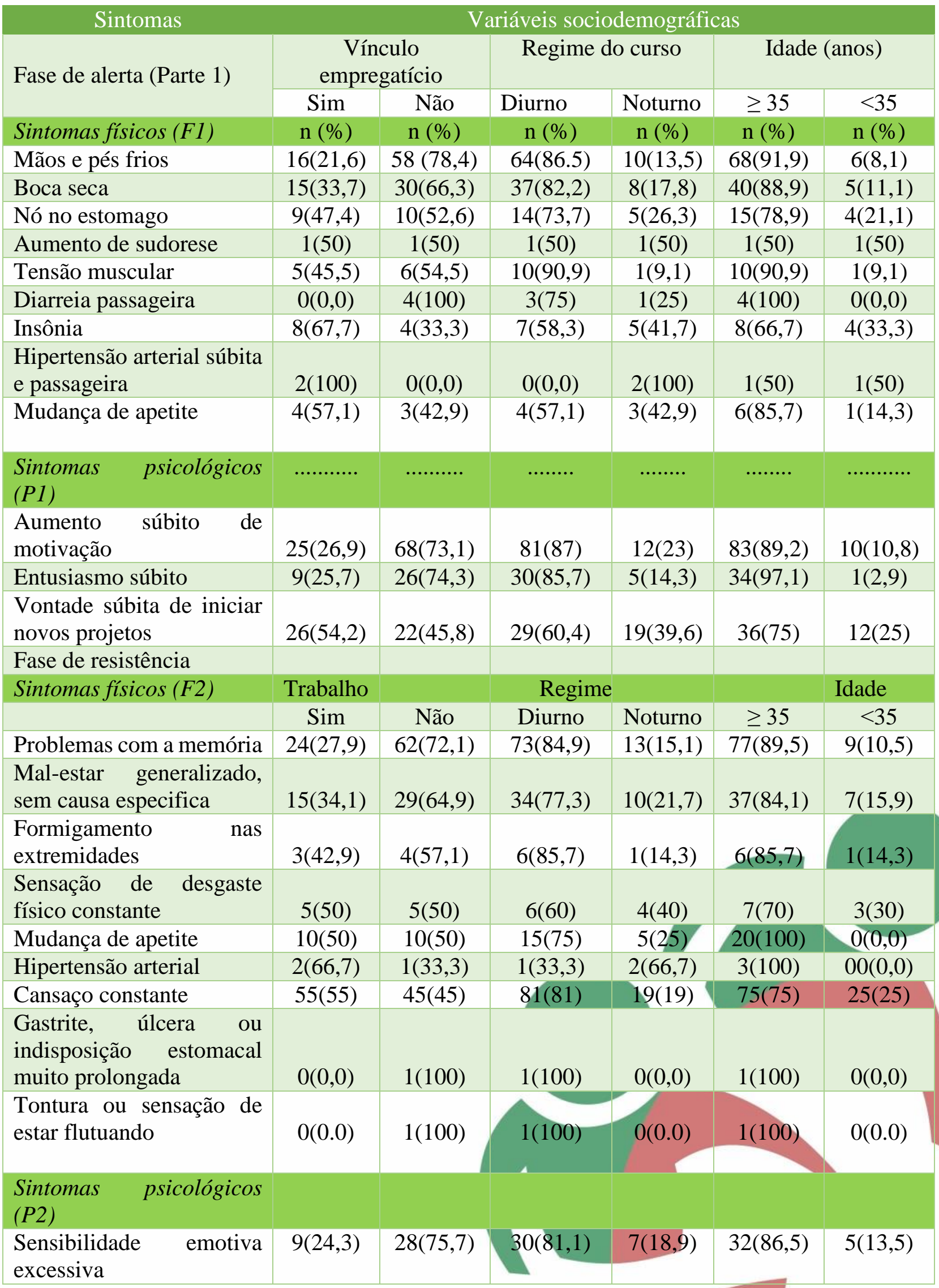




\begin{tabular}{|c|c|c|c|c|c|c|}
\hline Dúvida quanto a si próprio & $24(30)$ & $56(70)$ & $72(90)$ & $8(10)$ & $73(90,1)$ & $8(9,9)$ \\
\hline $\begin{array}{l}\text { Pensar constantemente em } \\
\text { um só assunto }\end{array}$ & $20(55,6)$ & $16(44,4)$ & $19(52,8)$ & $17(47,2)$ & $28(77,8)$ & $8(22,2)$ \\
\hline Irritação excessiva & $7(31,8)$ & $15(68,2)$ & $18(81,8)$ & $4(8,2)$ & $20(90,9)$ & $2(9,1)$ \\
\hline Diminuição da libido & $0(0,0)$ & $1(100)$ & $1(100)$ & $0(0,0)$ & $1(100)$ & $0(0,0)$ \\
\hline \multicolumn{7}{|l|}{ Fase de exaustão (Parte 3 ) } \\
\hline Sintomas físicos (F3) & Sim & Não & Diurno & Noturno & $\geq 35$ anos & $<35$ anos \\
\hline Diarreia frequente & $5(35,7)$ & $9(64,3)$ & $11(78,6)$ & $3(21,4)$ & $13(92,8)$ & $1(7,2)$ \\
\hline Dificuldades sexuais & $11(31,4)$ & $24(68,6)$ & $31(88,6)$ & $4(11,4)$ & $30(85,7)$ & $5(14,3)$ \\
\hline Insônia & $22(37,9)$ & $36(62,1)$ & $43(74,1)$ & $15(25,9)$ & $47(81,0)$ & $11(9,0)$ \\
\hline Náusea & 4 & $11(73,3)$ & $13(86,7)$ & $2(13,3)$ & $13(86,6)$ & $2(13,4)$ \\
\hline Tiques & $2(50)$ & $2(50)$ & $3(75)$ & $1(25)$ & $4(100)$ & $0(0.0)$ \\
\hline $\begin{array}{l}\text { Hipertensão } \\
\text { continuada }\end{array}$ & $5(71,4)$ & $2(28,6)$ & $3(42,9)$ & $4(57,1)$ & $4(57,1)$ & $3(42,9)$ \\
\hline $\begin{array}{l}\text { Problemas } \\
\text { dermatológicos } \\
\text { prolongados }\end{array}$ & $0(0.0)$ & $7(100)$ & $7(100)$ & $0(0.0)$ & $7(100)$ & $0(0,0)$ \\
\hline $\begin{array}{l}\text { Mudança extrema de } \\
\text { apetite }\end{array}$ & $9(33,3)$ & $18(66,7)$ & $20(74,1)$ & $7(25,9)$ & $26(96,2)$ & $1(3,8)$ \\
\hline Excesso de gases & $2(40,0)$ & $3(60,0)$ & $5(100)$ & $0(0,0)$ & $5(100)$ & $0(0,0)$ \\
\hline Tontura frequente & $0(0.0)$ & $3(100)$ & $3(100)$ & $0(0,0)$ & $3(100)$ & $0(0,0)$ \\
\hline Infarto & $0(0.0)$ & $1(100)$ & $1(100)$ & $0(0.0)$ & $1(100)$ & $0(0,0)$ \\
\hline $\begin{array}{l}\text { Sintomas psicológicos } \\
(P 3)\end{array}$ & Sim & Não & Diurno & Noturno & $\geq 35$ anos & $<35$ anos \\
\hline $\begin{array}{l}\text { Impossibilidade de } \\
\text { trabalhar }\end{array}$ & $13(33,3)$ & $26(66,7)$ & $31(79,5)$ & $8(20,5)$ & $34(87,1)$ & $5(12,9)$ \\
\hline Pesadelos frequentes & $20(37,7)$ & $33(62,3)$ & $42(79,2)$ & $11(20,8)$ & $47(88,6)$ & $6(11,4)$ \\
\hline $\begin{array}{l}\text { Sensação } \\
\text { incompetência em } \\
\text { as áreas }\end{array}$ & $2(14,3)$ & $12(85,7)$ & $14(100)$ & $0(0,0)$ & $13(92,8)$ & $1(7,2)$ \\
\hline Vontade de fugir de tudo & $5(25)$ & $15(75)$ & $16(80)$ & $4(20)$ & $9(90)$ & $1(10)$ \\
\hline $\begin{array}{l}\text { Apatia, depressão ou raiva } \\
\text { prolongada }\end{array}$ & $2(40)$ & $3(60)$ & $3(60)$ & $2(40)$ & $4(80)$ & $1(20)$ \\
\hline $\begin{array}{l}\text { Cansaço } \\
\text { excessivo }\end{array}$ & $7(36,8)$ & $12(63,2)$ & $15(78,9$ & $4(21,1)$ & $16(84,2)$ & $3(15,8)$ \\
\hline $\begin{array}{l}\text { Pensar e falar } \\
\text { constantemente em um só } \\
\text { assunto }\end{array}$ & $8(40)$ & $12(60)$ & $13(65)$ & $7(35)$ & $15(75)$ & $5(25)$ \\
\hline $\begin{array}{l}\text { Irritabilidade frequente } \\
\text { sem causa aparente }\end{array}$ & $1(50)$ & $1(50)$ & $2(100)$ & $0(0,0)$ & $2(100)$ & $0(0.0)$ \\
\hline $\begin{array}{l}\text { Angustia, ansiedade e } \\
\text { medo diariamente }\end{array}$ & $1(50)$ & $1(50)$ & $2(100)$ & $0(0,0)$ & $2(100)$ & $0(0,0)$ \\
\hline $\begin{array}{l}\text { Hipersensibilidade } \\
\text { emotiva }\end{array}$ & $1(50)$ & $1(50)$ & $2(100)$ & $0(0,0)$ & $1(50)$ & $1(50)$ \\
\hline
\end{tabular}

Fonte: Autores 
A leitura das Tabelas 2 e 3, permite visualizar que os estudantes de sexo masculino, solteiros, do primeiro ano do curso, desempregados, do curso diurno e mais novos têm maiores chances de sofrer o stress, pois pontuaram mais sinais desse fenômeno sobretudo na de resistência.

\section{Fases do stress nos estudantes}

Adicionalmente, foram realizadas análises visando identificar as fases de stress. O Gráfico 1 apresenta os resultados obtidos para cada fase da doença (alarme, resistência e exaustão).

Grafico 1: Fase de stress nos estudantes $(\mathrm{n}=176)$

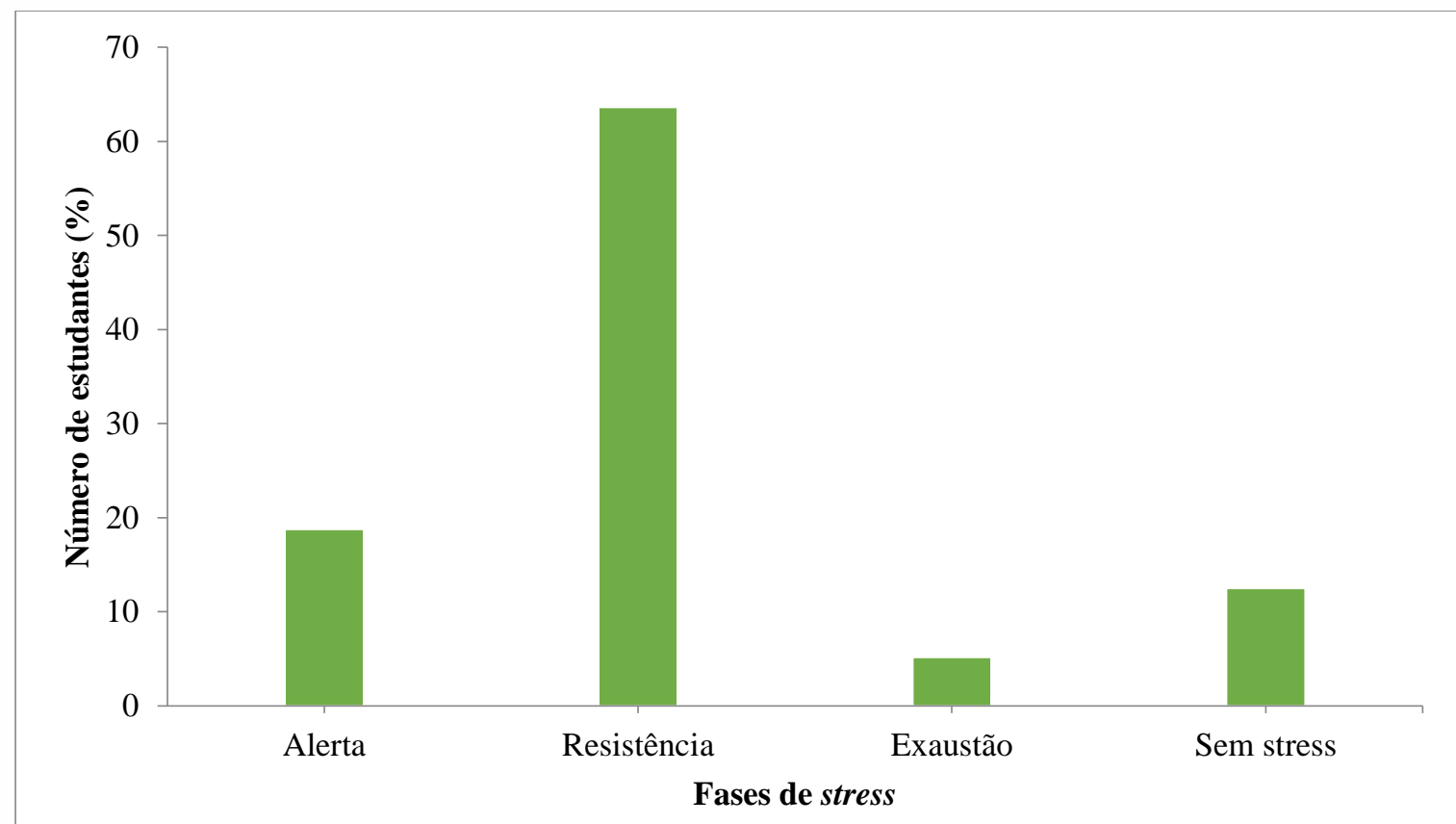

Fonte: Autores

O Gráfico 1 mostra que um total de 87,5\% (n=154) apresenta sintomas de stress, sendo que $18,75 \%(n=33)$ se enquadram na fase de alerta, $63,6 \%(n=112)$ na de resistência e, $5,15 \%$ $(n=9)$ na exaustão. Estes resultados mostram que mais da metade dos participantes se encontra a desenvolver sintomatologia da fase de resistência. 


\section{DISCUSSÃO}

A análise dos resultados indica que $87,5 \%$ dos alunos pesquisados apresentaram stress, sendo que a maioria 63,6\% está na fase de resistência, com predominância de sintomas de natureza psicológica. A taxa de incidência de stress obtida neste estudo é superior a de outros estudos com amostras de estudantes do ensino superior. Um estudo de Querido et al. (2016) verificou que $52 \%$ da amostra estudada apresentava stress com maior frequência de sintomas psicológicos. Na mesma direção, outros autores (e.g., Lima et al. 2016; Estrela, Rezende, Guedes, Pereira, \& Souza, 2018; Vieira \& Schermann, 2015; Cestari et al.,2017) que usaram o ISSL na avaliação dos níveis de stress em estudantes universitários acharam uma incidência da doença na ordem de 60,09\%; 61,59\%; 63,3\% e 64\% respectivamente. Também Assis et al. (2013) e Costa et al. (2018) identificaram níveis de incidência de stress em estudantes sucessivamente de $71 \%$ e 78,98\%. No que se refere à fase predominante, Chaves et al. (2016) e Costa et al. (2018) constaram que a maioria da amostra envolvida nos seus estudos ( $n=71)$ e $(n=155)$ se encontravam na fase de resistência. No já citado estudo de Costa et al. (2018) verificou-se o predomínio de sintomas psicológicos (42,0\%), na fase de resistência.

Uma revisão de literatura de Benavente e Costa (2011), concluiu que dos 13 artigos analisados, a maioria apontava a frequência de sintomas psicológicos, ou seja, cinco abordaram manifestações psicológicas, quatro manifestações fisiológicas e outros quatro estudos ambos os sintomas, o que encontra respaldo com os resultados obtidos neste estudo.

Em função das variáveis "sexo", "estado civil", "ano de freqüência", "vínculo empregatício", "regime do curso" e "idade" este estudo revelou que, de forma geral, ser do sexo masculino, solteiro, do primeiro ano do curso, desempregado, do curso diurno e mais novo constituem variáveis com maior chance de sofrer o stress, pois os sujeitos com essas características obtiveram mais pontuações nas diversas sintomatologias de stress, nas fases de alerta e resistência.

Hipoteticamente, existem cinco possíveis explicações para os achados desta pesquisa. A primeira prende-se com o fato de os estudantes dos cursos regulares (diurno) serem, na sua maioria jovens solteiros, sem vínculo empregatício. E como tal, estes podem sentir-se pressionados pela vida, no sentido de conseguirem melhor aproveitamento pedagógico para garantir melhores oportunidades profissionais futuras, $o$ que lhes exige um esforço adicional. Além do mais, a falta de vínculo empregatício faz com que os estudantes enfrentem dificuldades 
financeiras para custear os seus estudos, uma vez que em Moçambique não há políticas eficazes de assistência ao estudante.

A segunda explicação é o fato de os estudantes jovens viverem ainda na incerteza sobre o seu futuro profissional, quando comparado com os estudantes-trabalhadores que têm sua situação de emprego regularizada. A terceira é que os estudantes dos anos iniciais devem estar a enfrentar problemas de adaptação e integração acadêmica e/ou de lidar com as novas disciplinas e ambiente acadêmico, o que pode propiciar a ansiedade e o stress. A quarta pode estar relacionada com o fato de os estudantes viverem fora das suas famílias; vindo de outros locais distantes da cidade de Nampula, sendo que a falta de amparo pode constituir motivo de tensão psicológica. A quinta e última explicação é a de que, contrariamente aos estudantes mais novos (de regime diurno), os mais velhos, empregados e que se encontram no final do curso têm maior experiência para lidar com situações stressantes, um domínio maior do ambiente acadêmico e maiores expectativas de mudança de carreira e ingressarem no mercado de trabalho respectivamente.

Contrariamente aos achados desta pesquisa, o estudo de Cestari et al. (2017) constatou que os estudantes do sexo feminino, com companheiro e os do último ano da faculdade tiveram maiores chances de apresentar stress. Na mesma perspectiva, Estrela et al. (2018) observaram que as mulheres, os estudantes sem bolsa de estudo, as com menor satisfação com o curso tiveram maiores pontuações de stress.Outros estudos (e.g., Assis et al. 2013; Costa et al., 2018; Vieira \& Schermann, 2015), estão na mesma direção ao constatarem que o ser do sexo feminino foi uma variável preditora de maiores níveis de stress.

\section{CONSIDERAÇÕES FINAIS}

Este estudo teve como objetivo avaliar a sintomatologia de stress em estudantes de graduação de uma universidade pública da região norte de Moçambique. O principal mérito do estudo é o fato de ser o pioneiro na pesquisa sobre sintomas de stress numa amostra de estudantes do ensino superior em Moçambique. Os resultados alcançados sinalizam a presença de stress nos estudantes envolvidos na pesquisa, na fase de resistência. Adicionalmente, ilustram maior ocorrência de dez sintomas (mãos e pés frios; boca seca; aumento súbito de motivação; vontade súbita de iniciar novos projetos; entusiasmo súbito; problemas com a memória; mal-estar generalizado, sem causa especifica; cansaço constante; dúvida quanto a si próprio; insônia e pesadelos frequentes). 
As variáveis sociodemográficas e acadêmicas fazem diferença na ocorrência de stress nos estudantes. Os estudantes do sexo masculino, com idades compreendidas entre 17 e 35 anos, no início do curso, solteiros, do curso diurno e sem vínculo empregatício tendem a sofrer mais de stress no ambiente acadêmico.

Uma limitação óbvia deste estudo é ter sido realizado num contexto sociocultural singular e por não ter envolvido amostra probabilística, o que não permite generalizar os resultados para todos os estudantes moçambicanos. Nesse sentido, futuras pesquisas envolvendo amostras probabilísticas e diversificadas objetivando identificar os fatores de stress acadêmico e estratégias de enfrentamento são necessárias para melhor compreender o fenômeno estudado e propor medidas de prevenção e/ou intervenção, de modo a contribuir para a promoção da saúde mental nos estudantes moçambicanos.

\section{REFERÊNCIAS}

Anacleto Estrela Y. C., Costa Rezende, A. C., Ferreira Guedes, A., Oliveira Pereira C., Nunes, A. S. M. (2018). Stress e correlatos com características de saúde e sociodemográficas de estudantes de medicina. Rev. CES Med., 32(3), 215-225. doi: http://dx.doi.org/10.21615/cesmedicina.32.3.3

Araújo, K. J. S., Silva, A. M., Silva Júnior, G. M. N., Carrijo, J. B., Caixeta, L. F., \& Bagasra, A. (2016). Sofrimento mental: avaliação em uma universidade americana. Rev. Educ. Saúde, 4(2), 64-71. Recuperado de periodicos.unievangelica.edu.br/index.php/educacaoemsaude/article/view/2018

Ariño, D. O. \& Bardagi, M. P. (2018). Relação entre fatores acadêmicos e a Saúde Mental de estudantes universitários. Psicologia Pesquisa, 12(3), 44-52. http://dx.doi.org/10.24879/2018001200300544

Assis, C. L., Silva, A. P. F., Lopes, M. S., Silva, P. C. B., \& Santini, T. O. (2013). Sintomas de stress em concluintes do curso de psicologia de uma faculdade privada do norte do País. Mudanças - Psicologia da Saúde, 21(1), 23-28. doi: http://dx.doi.org/10.15603/21761019/mud.v21n1p23-28

Benavente, S. B. T. \& Costa, A. L. S. (2011). Respostas fisiológicas e emocionais ao stress em estudantes de enfermagem: revisão integrativa da literatura científica. Acta Paul. Enferm., 24(4), 571-6. http://dx.doi.org/10.1590/S0103-21002011000400019

Bublitz, S., Guido, L. A., Lopes, L. F. D., Freitas, E. O. (2016). Associação entre stress e características sociodemográficas e acadêmicas de estudantes de enfermagem. Texto Contexto Enferm., 25(4), 2-7.http://dx.doi.org/10.1590/0104-07072016002440015

Castro, V. R. (2017). Reflexões sobre a Saúde mental do estudante universitário: estudo empírico com estudantes de uma instituição pública de ensino superior. Revista Gestão em 
Foco, 9, 380-401. Recuperado em: portal.unisepe.com.br/unifia/wpcontent/uploads/sites/.../06/043_saude_mental.pdf

Cestari, V. R. F., Barbosa, I. V., Florêncio, R. S., Vera Lúcia Mendes de Paula Pessoa, V. L. M. P., \& Moreira, T. M. M. (2017). Stress em estudantes de enfermagem: estudo sobre vulnerabilidades sociodemográficas e acadêmicas. Acta Paul Enferm., 30(2), 190-6. http://dx.doi.org/10.1590/19820194201700029

Chaves, L. B., Souza, T. F., Silva, M. V. C., Oliveira, C. F., Lipp, M. E. N., Pinto, M. L. (2016). Stress em universitários: análisesanguínea e qualidade de vida. Revista Brasileira de Terapias Cognitivas, 12(1), 20-26. http://dx.doi.org/10.5935/1808-5687.20160004

Chagas, M. K. S., Moreira Junior, D. B., Cunha, G. N., Caixeta, R. P., \& Fonseca, E. F. (2016). Ocorrência da Síndrome de Burnout em acadêmicos de medicina de instituição de ensino no interior de Minas Gerais. Rev. Med. Saúde Brasília, 5(2), 234-245. Recuperado em: https://bdtd.ucb.br/index.php/rmsbr/article/view/7241

Costa, C. R. B., Maynart, W. H. C., Oliveira, L. B., Albuquerque, M. S. C., \& Correia, D. S. (2018-set./dez.). Stress entre estudantes de graduação em enfermagem: Associação decaracterísticas sociodemográficas e acadêmicas. Revista Saúde e Pesquisa, 11(3), 475482. doi: https://doi.org/10.17765/2176-9206.2018v11n3p475-482

Estrela, Y. C. A., Rezende, A. C. C. Guedes, A. F., Pereira, C. O., \& Sousa, M. N. A. (2018). Stress e correlatos com características de saúde e sociodemográficas de estudantes de medicina. Rev. CES Med., 32(3), 215-225. doi: http://dx.doi.org/10.21615/cesmedicina.32.3.3

Faiad, C., Souza, V., Matsunaga, L. H., Rodrigues, C. M. L., \& Rosa, H. R. (2018). Propriedades psicométricas do ISSL no contexto da segurança pública. Estudos Interdisciplinares em Psicologia, 9(3), 54-72. doi: http://dx.doi.org/10.5433/22366407.2018v9n3suplp54

Hirsch, C. D., Barlem, E. L. D., Almeida, L. K., Tomaschewski-Barlem, J. G., Lunardi, V. L., \& Ramos, A. M. (2018). Fatores percebidos pelos acadêmicos de enfermagem como desencadeadores do stress no ambiente formativo. Texto Contexto Enferm, 27(1), 1-11. http://dx.doi.org/10.1590/0104-07072018000370014

Lima, R. L., Soares, M. E. C., Prado, S. N., \& Albuquerque, G. S. C. (2016). Stress do estudante de medicina e rendimentoacadêmico. Rev. Brasil. de Ed. Médica, 40(4), 678-684. doi: http://dx.doi.org/10.1590/1981-52712015v40n4e01532015

Limongi-França, A. C. \& Rodrigues, A. L. (2009). Stress e trabalho: uma abordagem psicossomática, (4a . ed., 3. reimp.). São Paulo: Atlas

Lipp, M. E. N. (2000). Inventário de Sintomas do Stress para Adultos. São Paulo: Casa do Psicólogo.

Lipp. M. E. N. (2016). O Stress do professor frente ao mau comportamento do aluno. In D. C. Fava (Org.). A Prática da Psicologia na Escola (pp. 351-37). Belo Horizonte: Artesâ. 
Lipp, M. E. N., Costa, K. R. S. N., \& Nunes, V. O. (2017). Stress, qualidade de vida e estressores ocupacionais de policiais: Sintomas mais frequentes. Revista Psicologia: Organizações e Trabalho, 17(1), 46-53. http://dx.doi.org/10.17652/rpot/2017.1.12490.

Martins, C., Campos, S., Duarte, J., Martins, R., Moreira, T., \& Chaves, C. (2017). Situações indutoras de stress e Burnout em estudantes de enfermagem nos ensinos clínicos. Revista Portuguesa de Enfermagem de Saúde Mental, 5, 25-32. http://dx.doi.org/10.19131/rpesm.0163

Murphy, L. R. (2008). Mental Capital and Wellbeing: Making the most of ourselves in the $21^{\text {st }}$ century. The Government Office for Science.

Querido, I. A., Naghettini, A. V., Orsini, M. R. C. A.,Bartholomeu, D.,Montie, J. M. (2016). Fatores associados ao stress no internato médico. Rev. Bras. Educ. Med., 40(4), 565-573. http://dx.doi.org/10.1590/1981-52712015v40n4e00072015

Ramos, S. I. V. \& Carvalho, A. J. R. (2008). Nível de stresse e estratégias de coping dos estudantes do $1^{\circ}$ ano do ensino universitário de Coimbra. Psicologia, 1-17. Recuperado em: www.psicologia.com.pt

Salomão, I. F. C. S., Abacar, M., \& Aliante, G. (2018). Satisfação acadêmica em estudantes de ensino superior: Um estudo no curso de graduação em ensino básico da Universidade Pedagógica-Delegação de Nampula. Revista Brasileira de Ensino Superior, 4(1), 24-42. http://doi.org/10.18256/2447-3944.2018.v4i1.2239

Santos, J. A. F., Lucena, N. M. G., Rocha, T. V., Aragão, P. O. R. Gatto-Cardia, M. C., Carvalo, A. G. C., \& Barros, M. F. A. (2012). Stress em Acadêmicos do Curso de Fisioterapia. Revista Brasileira de Ciências da Saúde, 16(2), 89-94. Recuperado em: https://www.researchgate.net/publication/273272271_Stress_em_Academicos_do_Curso de_Fisioterapia

Souza, R. G., Santana, E. B., Pedra, R., Dias, D., Henrique, E., \& Dantas, M. (2015). A relevância dos instrumentos de avaliação de ansiedade, stress e depressão. Ciências Biológicas e de Saúde, 3(1), 37-57. Recuperado https://periodicos.set.edu.br/index.php/cadernobiologicas/article/view/2493

Vieira, L. N. \& Schermann, L. B. (2015). Stress e fatores associados em alunos de psicologia de uma universidade particular do sul do Brasil. Aletheia, 46, 120-130. Recuperado em: http://pepsic.bvsalud.org/scielo.php?script=sci_arttext\&pid=S1413-03942015000100010 